Radiologe 2021 · 61:909-914

https://doi.org/10.1007/s00117-021-00901-8

Angenommen: 29. Juli 2021

Online publiziert: 24. September 2021

(c) Springer Medizin Verlag GmbH, ein Teil von

Springer Nature 2021

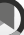

Check for
updates

\section{COVID-19-induzierte Koagulopathien und thromboembolische Manifestationen}

\author{
O. Sedlaczek ${ }^{1,2} \cdot$ W. Wagner ${ }^{2,3} \cdot$ C. E. Dempfle ${ }^{4}$ \\ ${ }^{1}$ Radiology Cooperation Uni/DKFZ, Division of Radiology, NCT, Heidelberg, Deutschland \\ ${ }^{2}$ Translational Lung Research Center, Member of the German Center for Lung Research, University of \\ Heidelberg, Heidelberg, Deutschland \\ ${ }^{3}$ Department of Diagnostic and Interventional Radiology, University of Heidelberg, Heidelberg \\ Deutschland \\ ${ }^{4}$ Coagulation Center Mannheim, Mannheim, Deutschland
}

\section{Zusammenfassung}

\section{In diesem Beitrag}

- Management der Antikoagulation oder der Gerinnungsstörung?

Klinisches Problem: Klinisch wird COVID-19 („corona virus disease“ 2019) zunehmend als systemische Erkrankung gesehen, in deren Zentrum eine Multiorganbeteiligung durch einen hyperkoagulatorischen Zustand im Sinne einer Vaskulopathie steht. Therapeutische Standardverfahren: Eine Behandlung mit Thrombozytenfunktions-

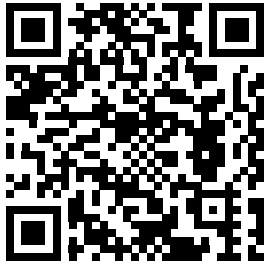

QR-Code scannen \& Beitrag online lesen hemmern oder Heparinen erscheint die logische Konsequenz. Die aktuelle Studienlage, zumindest für ASS, ergibt allerdings keine Hinweise auf eine Wirksamkeit.

Diagnostik: Entsprechend der zu nennenswerten Anteilen primär mikrostrukturellen Gefäßveränderungen sollte die radiologische Diagnostik nicht nur Makrogefäßpathologien, sondern auch Hinweise auf diffuse Perfusionsstörungen darstellen. Leistungsfähigkeit: In der Lunge sind beispielsweise Perfusionsausfälle nachweisbar, die nur teilweise durch pulmonalarterielle Füllungsdefekte zu erklären sind. Ähnliche Befunde zeigen sich in fast allen Organsystemen.

Empfehlung für die Praxis: Eine therapeutische Intervention mittels niedermolekularer Heparine bei hospitalisierten Patienten in situationsadaptierter Dosierung ist indiziert und wird ausführlich besprochen. Bei Nachweis von Mikro- und Makrogefäßthrombosierung im Rahmen von COVID-19 spielt die erweiterte radiologische Diagnostik eine zentrale Rolle und ist die Basis der Therapie und Sekundärprävention.

\title{
Schlüsselwörter
}

SARS-CoV-2 $\cdot$ Koagulopathie $\cdot$ Computertomographie $\cdot$ Perfusion $\cdot$ Therapie

Tiefe Beinvenenthrombosen, venöse Thromboembolien und Lungenarterienembolien treten bei mit COVID-19 (",corona virus disease 2019“) hospitalisierten Patienten gehäuft auf, insbesondere bei schwerem Verlauf. Thromboembolische Ereignisse haben bei $\mathrm{Pa}$ tienten mit schwerem COVID-19-Verlauf eine wesentlich höhere Mortalität als bei Patienten ohne COVID-19. Die radiologischen Befunde, insbesondere der Lunge, haben früh an ein vaskulitisches/ systemisches Geschehen denken lassen.
Der Einsatz niedermolekularer Heparine bzw. einer oralen Antikoagulation senkt das Risiko deutlich.

Frühen Studien zufolge erleiden etwa 20 \% der mit COVID 19 hospitalisierten Patienten einen akuten Lungenschaden (ARDS; [17]). In der weiteren Entwicklung der Pandemie fiel allerdings auf, dass ein Teil dieser Patienten nicht die für die ARDS klassische Erhöhung der pulmonalen Elastance entwickelte [5]. Die Beobachtung einer gesteigerten pulmonalen Angiogenese ge- 


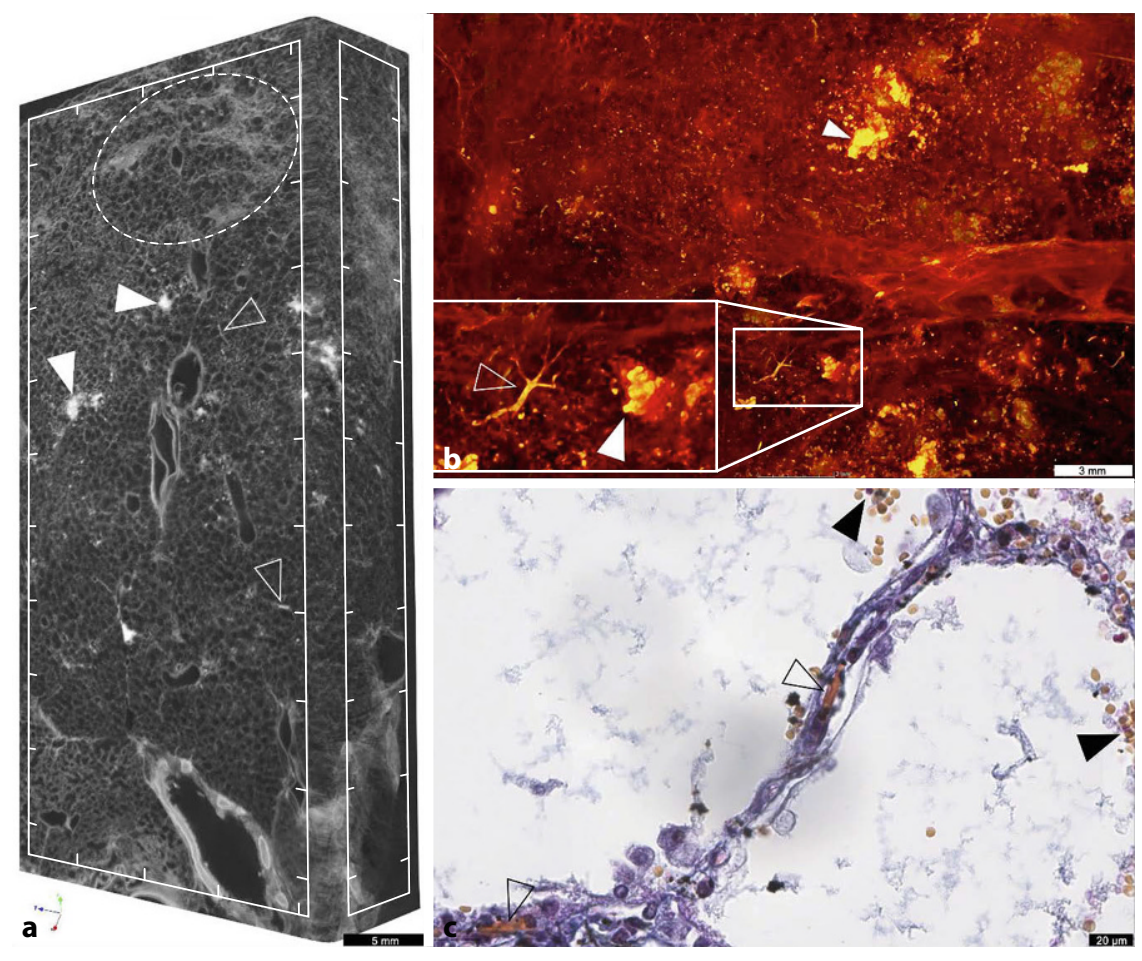

Abb. 1 \ Alveoläre Mikrothrombose und fokale alveoläre Hämorrhagie bei COVID-19. a Mikro-Computertomographie ( $\mu \mathrm{CT}$ ) eines Lungenpräparats eines 71-jährigen COVID-19-Patienten nach invasiver Beatmung und Vollheparinisierung mit Nachweis von alveolären Mikrothromben (offene Pfeile), fokaler alveolärer Hämorrhagie (Pfeile) und beginnenden peripheren Fibrosierungen (Oval). b Volumenrendering der alveolären Mikrothromben (offene Pfeile) und der fokalen alveolären Hämorrhagie (Pfeile). c Histopathologischer Nachweis fibrinoider kapillarer Mikrothromben (offene Pfeile) und fokaler alveolärer Hämorrhagie (Pfeile). a, b Nach chemischer Trocknung des Lungengewebes erfolgte die Bildakquisition mittels Bruker SkyScan $1176 \mu \mathrm{CT}$ mit einer Voxelkantenlänge von $9 \mu \mathrm{m}$. Für die graphische Darstellung wurden CTVox3.1.1 (Bruker Biospin, Rheinstetten, Deutschland;a) und VGSTUDIO Max 3.4.0 (Volume Graphics, Heidelberg, Deutschland; b) genutzt. c Fuchsin-Orange-G-Färbung einer direkt an die für $\mathbf{a}, \mathbf{b}$ verwendeten Region angrenzenden Gewebeprobe

paart mit einem erhöhten intrapulmonalen Rechts-zu-Links-Shunt veranlasste einige Autoren, das Akronym „AVDS“ für akutes vaskuläres Distresssyndrom zu prägen [8].

Eine Verbindung der SARS-CoV-2-Infektion mit thromboembolischen Ereignissen war eines der ersten als gesichert geltenden Kriterien zur klinischen Unterscheidung von der normalen Grippe und basierte auf Obduktionen an COVID-19 verstorbener Patienten [18]. Neben Thrombosen und Thrombembolien häuften sich im Verlauf bei den Obduktionsstudien vor allem Nachweise von Mikrothromben und einer Endothelialitis [1]. COVID-19 hat sich zunehmend als systemische Erkrankung herausgestellt, die von einer Fehlregulation des Immunsystems mit Hyperkoagulopathie gekennzeichnet ist. Bereits zu Beginn der Pandemie war der Nachweis auffälliger Gerinnungsparameter [15] als ungüns- tiger Prognoseparameter erkannt worden. Hierbei scheint ein erhöhtes D-Dimer ein verlässlicher prognostischer Indikator zu sein. Weitere Risikofaktoren sind Adipositas und ein erhöhter IL-6-Spiegel.

Mit zunehmendem Verständnis der COVID-19-Erkrankung als Vaskulopathie [15] rundet sich auch das Bild einer verthrombembolischen Ereignissen ab. Der Bildgebung kommt hierbei eine essenzielle Rolle in der Detektion vaskulärer

Bei schwer kranken COVID-19-Patienten war das progressive Lungenversagen deutlich häufiger mit dem pathologischen Nachweis thrombotischen Materials [18] vergesellschaftet als bei der Autopsie von Patienten mit Influenza A [3]. Auch venöse thrombotische Ereignisse (tiefe Venenthrombosen, Lungenarterienembolien [LAE]) werden bei Influenza wesentmehrten Entstehung von endothelseitigen Erkrankungsmanifestationen zu. lich seltener beobachtet als bei COVID19 [11]. In der Bildgebung fanden sich Thrombosen und Embolien bei $37 \%$ der intensivmedizinisch behandelten Patienten und bei $10 \%$ der COVID-19-Patienten auf Normalstationen [19].

Die vermehrte Thrombenbildung betrifft bei COVID-19-Patienten auch die kleinen Gefäße: Mikrovaskuläre Thrombosen waren bei COVID-19 neunmal so häufig wie bei Patienten mit Influenza [1]. Ramacciotti et al. fanden thromboembolische Ereignisse bei $49 \%$ der untersuchten intensivmedizinisch betreuten Patienten mit COVID19 [14], wobei $87 \%$ der betroffenen Patienten Lungenembolien, aber nur $4 \%$ tiefe Venenthrombosen aufwiesen. Es ist daher sehr wahrscheinlich, dass es sich bei den beobachteten makro- und mikrovaskulären Thromben in der Lunge von COVID19-Patienten zu nennenswerten Anteilen nicht um Lungenembolien handelt, sondern dass sie vor Ort, in den Lungengefäßen entstanden sind.

Auch wenn bei Patienten mit septischen Zustandsbildern grundsätzlich von einer disseminierten Gerinnungsaktivierung auszugehen ist und durch Akute-PhaseReaktion und Immobilisation ein generelles Thromboserisiko besteht, spricht das Verteilungsmuster für eine pulmonale Manifestation eines vaskulitischen Geschehens.

Typisch für COVID-19 sind daher sehr häufige und ausgedehnte pulmonale mikro- und makrovaskuläre Thrombosen, während die Inzidenz venöser Thrombosen (Beinvenenthrombosen, Armvenenthrombosen, aber auch intrakranieller Thrombosen) ähnlich ist wie bei Patienten mit anderen septischen Zustandsbildern. Die bildgebende Diagnostik sollte sich daher auf eine bestmögliche Beurteilung der pulmonalen Gefäßsituation konzentrieren (wenn möglich, unter Einschluss der Lungenperipherie und der Mikrozirkulation), während ein generelles Screening auf venöse Thrombosen nicht vordringlich ist.

Der Einsatz der Computertomographie (CT) zum Nachweis von COVID-19 anhand des charakteristischen Musters einer Viruspneumonie wurde rasch zugunsten der RT-PCR (Reverse-transcription-Polymerasekettenreaktion) verlassen. Nicht nur der Strahlenschutz spricht dagegen (s. SSK- 

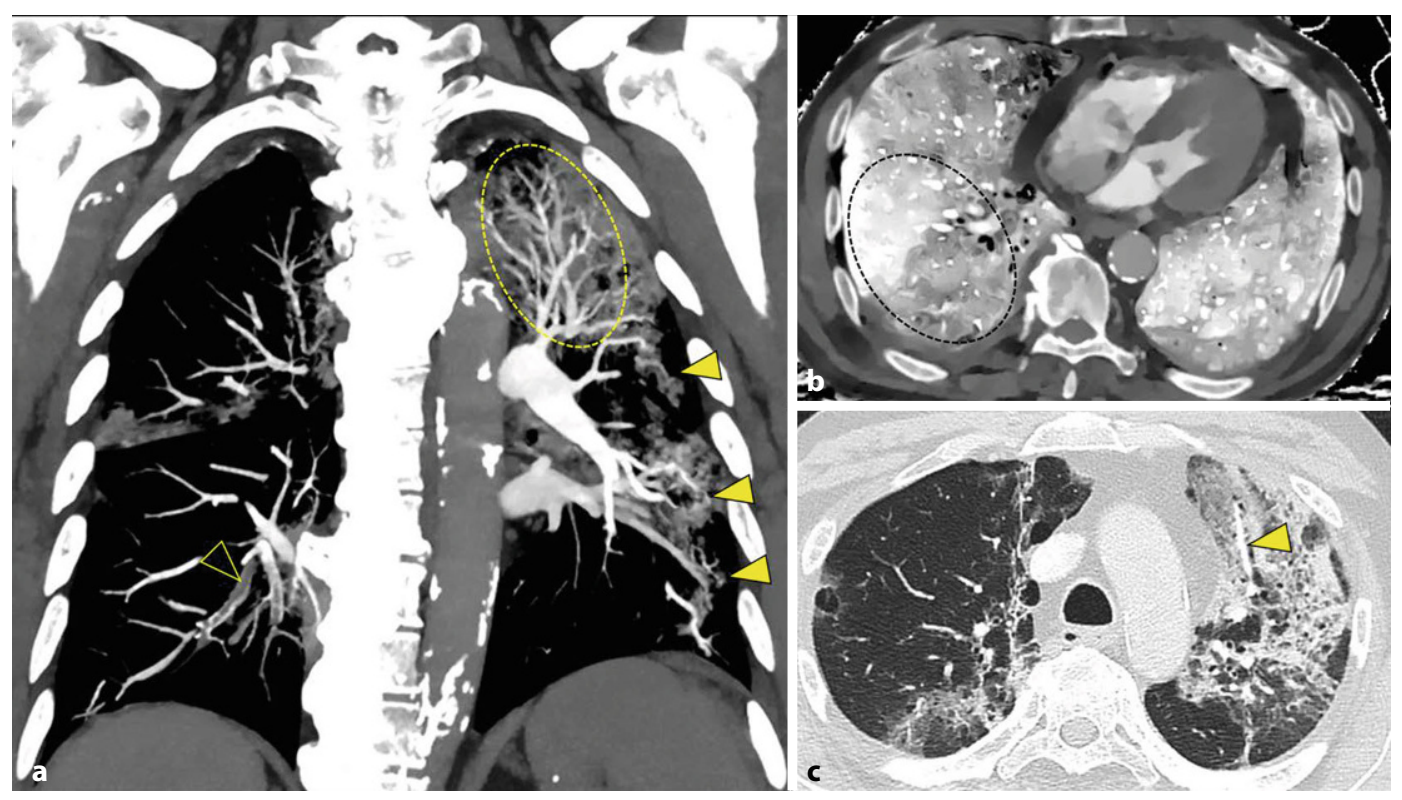

Abb. 2 \ Non-Makro-Lungenarterienembolie (LAE), pulmonal-vaskuläre Manifestationen im Rahmen von COVID-19.21-jährige Patientin mit COVID-19 und V. a. LAE. a Vereinzelte Milchglasinfiltrate v. a. in der Lungenperipherie (offener Pfeil), deutliche Dilatation peripherer Lungengefäße (geschlossene Pfeile). b Kein Nachweis einer LAE. c Deutliche Perfusionsinhomogenitäten in der Z Effective Karte mit milder Hypoperfusion der Milchglasinfiltrate (offener Pfeil), deutlicher Hyperperfusion von subpleuralen Arealen distal der dilatierten Lungengefäße (geschlossene Pfeile) und Perfusionsdefiziten in Arealen ohne Infiltrate oder makroskopischen Gefäßanomalien (Oval); siehe hierzu auch die Gefäßdarstellung in der Jodkarte (d). DualLayer-CT-System mit 64 Detektorzeilen (iQon Spectral CT, Philips Healthcare, Best, Niederlande). Zur Kontrastverstärkung wurde ein Hochdruckinjektor verwendet, um ein Kontrastmittelvolumen von $95 \mathrm{ml}$ (Accupaque $350 \mathrm{mgl} / \mathrm{ml}$; GE Healthcare, Braunschweig, Deutschland) mit einer Flussrate $\geq 3,0 \mathrm{ml} / \mathrm{s}$ zu injizieren

Stellungnahme in diesem Heft), sondern auch die mögliche Infektionsübertragung auf andere Patienten und das Personal.

Wenn heute CT-Untersuchungen angefordert werden, stehen Fragestellungen nach Sekundärkomplikationen und Differenzialdiagnosen im Vordergrund, insbesondere bei Patienten mit schwerer Symptomatik oder einer klinischen Verschlechterung [2]. Zu den relevanten Fragestellungen zählen das Ausmaß der entzündlichen Veränderungen der Lunge, der Nachweis einer möglichen Superbzw. Zweitinfektion und die Abgrenzeng diffuser (am ehesten mikrovaskulärer/ mikrothrombotischer) Veränderungen (- Abb. 1 und 2; [16]) von einer Lungenarterienembolie (- Abb. 3.). Die konkreten A-priori-Wahrscheinlichkeiten für das Vorliegen der entsprechenden Pathologie unterscheiden sich bei Patienten mit schwerem COVID-19-Verlauf deutlich vom intensivmedizinisch behandelten Influenzapatienten $[9,12]$. Die Prävalenz einer LAE beträgt $8 \%$ bei Intensivpatienten mit Influenza, $18 \%$ bei hospitalisierten COVID19-Patienten und $22 \%$ bei Patienten mit schwerem COVID-19-Verlauf.
Während die Inzidenz der akuten zerebralen Ischämie sich durch eine SARSCoV-2-Infektion nur moderat erhöht und im Wesentlichen den präexistenten Risikofaktoren zu folgen scheint [13], ist die Beteiligung abdominaler Gefäße überraschend häufig, und zwar meist in Form einer mikrovaskulären Beteiligung und Endorganischämie von Darm, Milz, Nieren, Leber oder Gallenblase [4]. Hierbei sind analog zur Lungenarterienembolie nur teilweise Makroverschlüsse der Arterien oder eine Portalvenenthrombose nachweisbar (•Abb. 4).

\section{Management der Antikoagulation oder der Gerinnungsstörung?}

Die Thrombozytenfunktionshemmung scheint in der bislang nur als pre-print erschienenen RECOVERY-Studie [6] für hospitalisierte Patienten ohne Wirksamkeit.

Niedermolekulare Heparine und Fondaparinux zeigen eine zuverlässigere gerinnungshemmende Wirkung, sind allerdings ebenfalls auf das bei schwer kranken Patienten häufig verminderte Antithrombin angewiesen. Bei niedermolekularem Heparin ist das Risiko einer HIT-2 erheblich geringer als bei Verwendung von unfraktioniertem Heparin; bei Fondaparinux ist nicht mit dem Auftreten einer HIT-2 zu rechnen. Nachteil von Fondaparinux ist allerdings die rein renale Elimination, die bei intensivmedizinischen Patienten mit stark eingeschränkter Nierenfunktion problematisch sein könnte.

Günstiger könnten direkt wirksame Antikoagulanzien sein, insbesondere Apixaban und Rivaroxaban, der Einsatz kann aber bisher wegen noch nicht vorhandener Studienergebnisse und fehlender $\mathrm{Zu}$ lassung zur Thromboseprophylaxe in dieser Indikation nicht empfohlen werden.

Die aktuellen Empfehlungen sehen vor, dass hospitalisierte Patienten mit SARS-CoV-2-Infektion eine medikamentöse Thromboseprophylaxe erhalten sollten, wobei für Patienten mit besonderem Risiko (bekannte Thromboseneigung, frühere thrombotische Ereignisse, eingeschränkte Mobilität, hohes Körpergewicht, aktive Tumorerkrankung, besondere vaskuläre Risikofaktoren wie Diabetes mellitus etc.) eine erhöhte Dosierung verwendet 

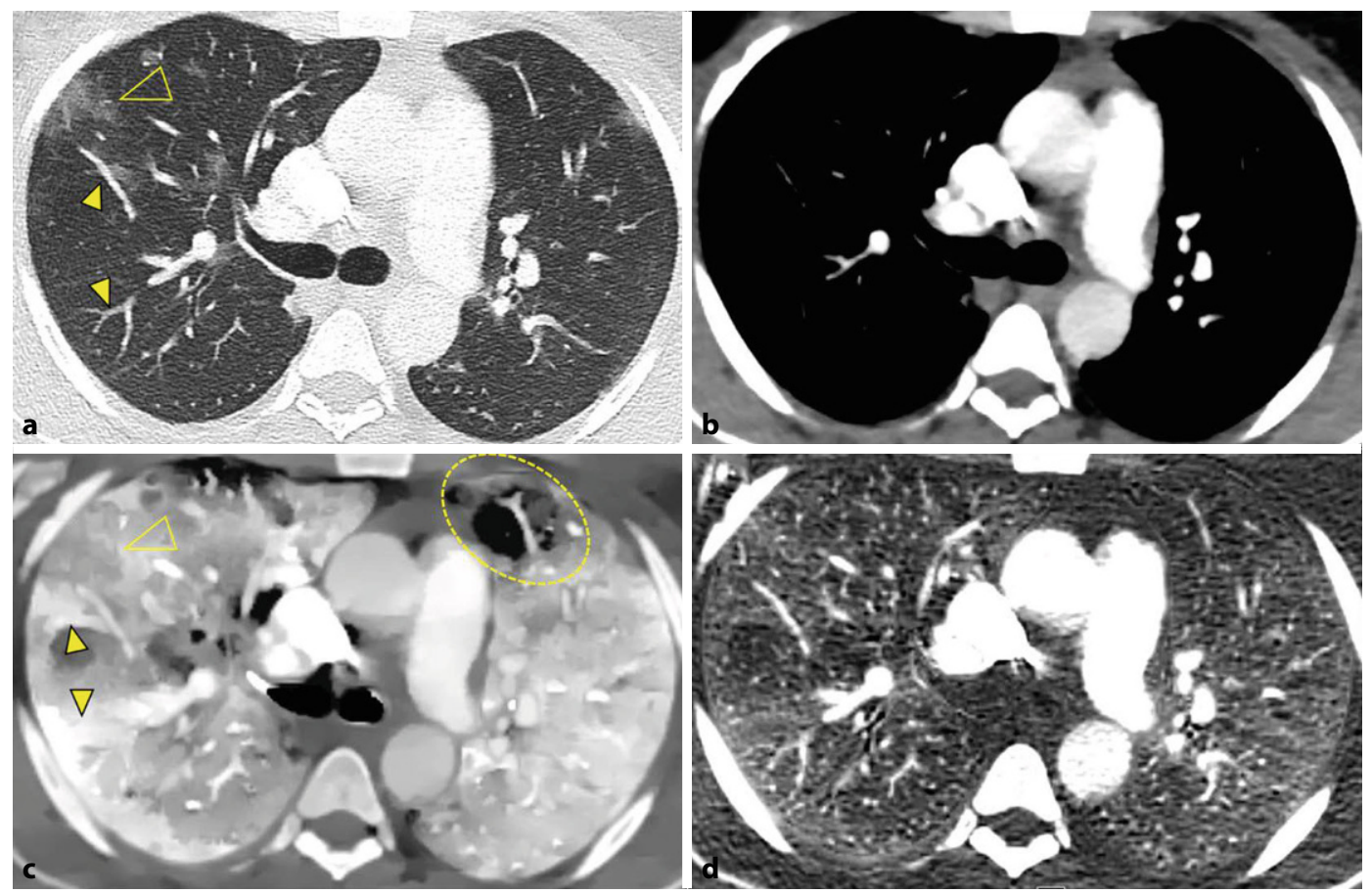

Abb. 3 ム Lungenarterienembolie (LAE) im Rahmen von COVID-19. 68-jähriger COVID-19-Patient unter Vollheparinisierung. Nach 3-wöchigem oligosymptomatischem Verlauf, neuaufgetretene Kollapsneigung, Schwindel und Bradykardien. Zu einem Vorbefund 7 Tage zuvor neu abgrenzbare LAEim rechten Unterlappen (offen Pfeile) mit entsprechenden Perfusionsausfällen in der Jodkarte (b; Oval) bei konstanten Infiltraten, betont im linken Oberlappen (gelbe Pfeile/Oval). Dual-Layer-CT-System mit 64 Detektorzeilen (iQon Spectral CT, Philips Healthcare, Best, Niederlande). Zur Kontrastverstärkung wurde ein Hochdruckinjektor verwendet, um ein Kontrastmittelvolumen von $95 \mathrm{ml}$ (Accupaque $350 \mathrm{mgl} / \mathrm{ml}$; GE Healthcare, Braunschweig, Deutschland) mit einer Flussrate $\geq 3,0 \mathrm{ml} / \mathrm{s}$ zu injizieren
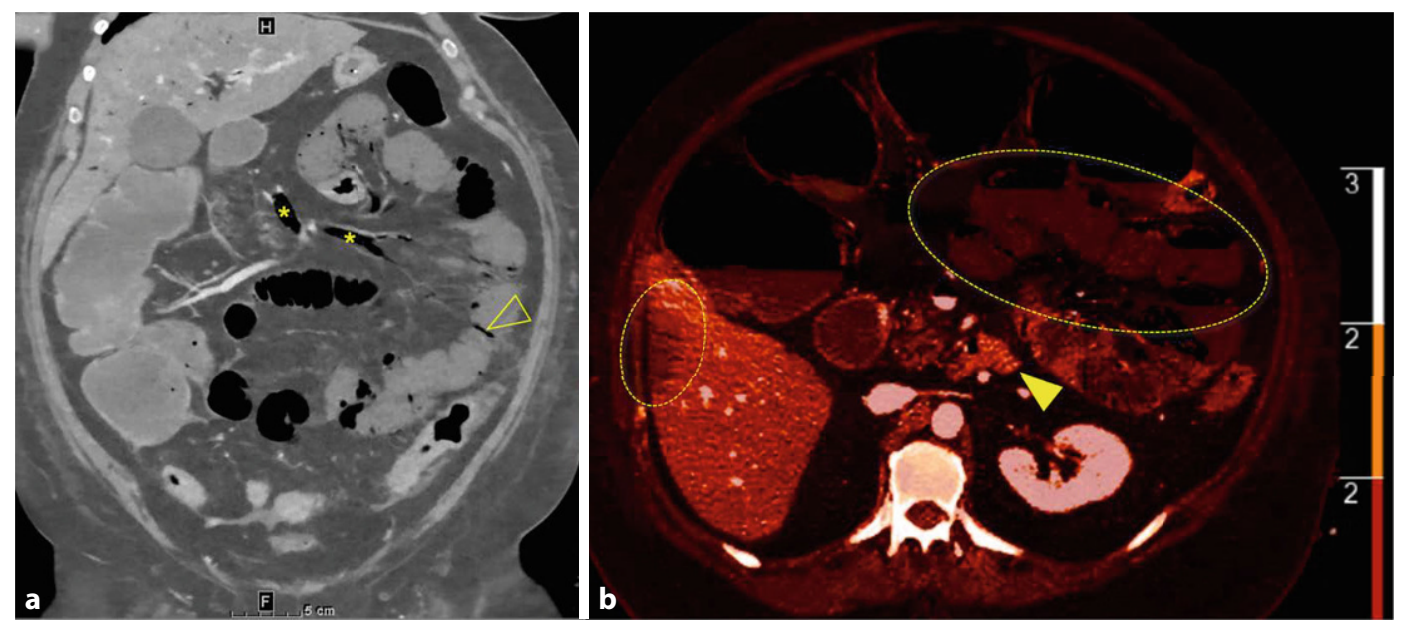

Abb. $4 \Delta$ Ausgedehnte Darmischämie im Rahmen von COVID-19. a Mesenterialischämie mit Pneumatosis intramuralis et portalis (offene Pfeile) ohne Nachweis proximaler Gefäßpathologien der A. mesenterica superior. In der Jodkarte kräftiger Kontrastierung der Duodenalwand und des proximalen Jejunums; im weiteren Verlauf bis Colon ascendens fehlend (gelbes Oval). Intraoperativ 4,8 m ischämischer Dünndarm, $60 \mathrm{~cm}$ Dickdarm. Histologisch Dünn- und Dickdarmischämie mit Innenschichtbetonten Nekrosen. b Dual-Layer-CT-System mit 64 Detektorzeilen (iQon Spectral CT, Philips Healthcare, Best, Niederlande). Zur Kontrastverstärkung wurde ein Hochdruckinjektor verwendet, um ein Kontrastmittelvolumen von $95 \mathrm{ml}$ (Accupaque $350 \mathrm{mgl} / \mathrm{ml}$; GE Healthcare, Braunschweig, Deutschland) mit einer Flussrate $\geq 3,0 \mathrm{ml} / \mathrm{s}$ zu injizieren 
wird [10]. Bisherige Untersuchungen mit niedermolekularem Heparin zeigen, dass eine therapeutische Dosierung wirksamer in der Verhinderung thrombotischer Ereignisse bei schwerer COVID-19 ist [7].

Enoxaparin, Dalteparin, Tinzaparin und Certoparin sowie Fondaparinux sind für die Thromboseprophylaxe bei nichtchirurgischen Patienten zugelassen, und es liegen eindeutige Empfehlungen für die Dosierung bei Patienten mit eingeschränkter Nierenfunktion vor.

Enoxaparin, Dalteparin, Tinzaparin und Certoparin können auch bei terminaler Niereninsuffizienz und Hämodialyse eingesetzt werden. Eine eingeschränkte Nierenfunktion ist daher kein Grund für die Verwendung von unfraktioniertem Heparin bei intensivmedizinischen Patienten allgemein und bei Patienten mit COVID-19 im Speziellen.

Nadroparin ist für die Thromboseprophylaxe bei nichtchirurgischen Patienten nicht zugelassen. Bei Nachweis einer Thrombose oder Embolie (also auch der o.g. pulmonalen mikro- und makrovaskulären Thromben) kann die für die Behandlung der tiefen Venenthrombose und/oder Lungenembolie übliche gerinnungshemmende Therapie erfolgen. Die aktuellen Leitlinien empfehlen eindeutig, niedermolekulare Heparine oder Fondaparinux gegenüber unfraktioniertem Heparin zu bevorzugen, und, wenn klinisch möglich, direkte orale Antikoagulanzien einzusetzen. Zu beachten ist, dass bei Verwendung von Edoxaban und Dabigatran eine Initialphase von 5-7 Tagen mit parenteraler Antikoagulation (bevorzugt niedermolekulares Heparin oder Fondaparinux) vorgesehen ist, während bei Verwendung von Rivaroxaban und Apixaban direkt mit der oralen Medikation gestartet wird. Bei Rivaroxaban ist eine erhöhte Initialdosis (2-mal $15 \mathrm{mg}$ für 3 Wochen, weitere Therapie mit 1-mal $20 \mathrm{mg}$ täglich) vorgesehen, ebenso bei Apixaban (2-mal $10 \mathrm{mg}$ für 1 Woche, dann 2-mal $5 \mathrm{mg}$ täglich). Vorteil von Apixaban ist, dass die Pharmakodynamik durch die Nierenfunktion nicht relevant beeinflusst wird und auch bei schwerer Nierenfunktionsstörung mit normaler Dosierung behandelt werden kann. Bei Rivaroxaban und Edoxaban ist hingegen bei Nierenfunktionsstörung eine Dosisre-

\section{COVID-19-induced coagulopathy and thrombosis manifestations}

Clinical issue: Clinically, COVID-19 (coronavirus disease 2019) is increasingly seen as a systemic disease associated with multiorgan involvement through a hypercoagulatory condition in the sense of vasculopathy.

Standard treatment: Treatment with antiplatelet drugs or heparins appears to be indicated. The current evidence, at least for acetylsalicylic acid (ASA), is lacking. Diagnostic work-up: Corresponding to the significant proportion of primarily microstructural vascular changes, the radiological diagnosis showed not only macrovascular pathologies, but also diffuse perfusion disorders.

Performance: Regional hypoperfusion in the lungs can be detected with and without pulmonary arterial embolism. Similar findings can be found in almost all organ systems. Practical recommendations: A therapeutic intervention using low molecular weight heparins in hospitalized patients in situation-adapted dosage is indicated and is discussed in detail. In the detection of micro- and macrovascular thrombosis in the context of COVID-19, extended radiological diagnostics play a central role and are the basis of adapted therapy and secondary prevention.

\section{Keywords}

SARS-CoV-2 · Coagulopathy · Computedtomography $\cdot$ Perfusion · Treatment

duktion erforderlich, und der Einsatz wird bei schwerer Nierenfunktionseinschränkung nicht empfohlen. Dabigatran ist bei stark eingeschränkter Nierenfunktion kontraindiziert, ebenso Phenprocoumon und Warfarin.

\section{Fazit für die Praxis}

- Wie in vielen klinischen Fächern ist COVID19 auch radiologisch ein sich in mannigfaltigen Formen manifestierendes Krankheitsbild.

- Bezüglich der diagnostischen Morphologie stehen sowohl pulmonal als auch im übrigen Körper lokal vaskulitisch verteilte thrombotische und nur teils thrombembolische Manifestationen im Vordergrund.

- Zur Darstellung der Pathologien sollte deshalb nicht nur auf den Nachweis des vermuteten Makrogefäßverschlusses geachtet werden, sondern auch mögliche Mikrogefäßparameter wie Kontrastdynamiken und somit der eigentlichen Perfusion.

- Mit der geeigneten Prophylaxe lassen sich die Verläufe deutlich positiv beeinflussen.
Korrespondenzadresse

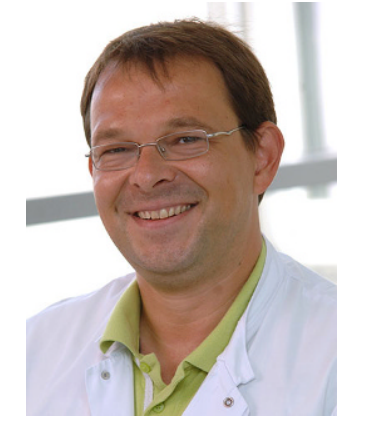

Dr. med. 0. Sedlaczek

Radiology Cooperation Uni/DKFZ, Division of Radiology, NCT

Im Neuenheimer Feld 460, 69120 Heidelberg, Deutschland

o.sedlaczek@med.uni-heidelberg.de

\section{Einhaltung ethischer Richtlinien}

Interessenkonflikt. O. Sedlaczek, W. Wagner und C.E. Dempfle geben an, dass kein Interessenkonflikt besteht.

Für diesen Beitrag wurden von den Autoren keine Studien an Menschen oder Tieren durchgeführt. Für die aufgeführten Studien gelten die jeweils dort angegebenen ethischen Richtlinien.

\section{Literatur}

1. Ackermann M, Verleden SE, Kuehnel M et al (2020) Pulmonary vascular endothelialitis, thrombosis, and angiogenesis in Covid-19. N Engl J Med 383:120-128

2. Akl EA, Blazic l, Yaacoub S et al (2021) Use of chest imaging in the diagnosis and management of 


\section{Leitthema}

COVID-19: a WHO rapid advice guide. Radiology 298:E63-E69

3. Burkhard-Koren NM, Haberecker M, Maccio U et al (2021) Higher prevalence of pulmonary macrothrombi in SARS-CoV-2 than in influenza A: autopsy results from "Spanish flu" 1918/1919 in Switzerland to Coronavirus disease 2019. J Pathol Clin Res 7:135-143

4. Caruso D, Zerunian M, Pucciarelli F et al (2021) Imaging of abdominal complications of COVID-19. Infection 3:20200052

5. Gattinoni L, Chiumello D, Caironi P et al (2020) COVID-19 pneumonia: different respiratory treatments for different phenotypes? Intensive Care Med 46:1099-1102

6. Group RC, Horby PW, Pessoa-Amorim G et al (2021) Aspirin in patients admitted to hospital with COVID-19 (RECOVERY): a randomised, controlled, open-label. platform trial.2021.2006.2008.21258132

7. Helms J, Severac F, Merdji H et al (2021) Higher anticoagulation targets and risk of thrombotic events in severe COVID-19 patients: bi-center cohort study. Ann Intensive Care 11:14

8. Jounieaux V, Rodenstein DO, Mahjoub Y (2020) On happy hypoxia and on sadly ignored "acute vascular distress syndrome" in patients with COVID-19. Am JRespir Crit Care Med 202:1598-1599

9. Liu Y, Cai J, Wang C et al (2021) Incidence, prognosis, and laboratory indicators of venous thromboembolism in hospitalized patients with coronavirus disease 2019: a systematic review and meta-analysis. J Vasc Surg Venous Lymphat Disord 9(5):1099-1111

10. Marietta M, Ageno W, Artoni A et al (2020) COVID19 and haemostasis: a position paper from Italian Society on Thrombosis and Haemostasis (SISET). Blood Transfus 18:167-169

11. Piroth L, Cottenet J, Mariet AS et al (2021) Comparison of the characteristics, morbidity, and mortality of COVID-19 and seasonal influenza: a nationwide, population-based retrospective cohort study. Lancet Respir Med 9:251-259

12. Poissy J, Goutay J, Caplan M et al (2020) Pulmonary embolism in patients with COVID-19: awareness of an increased prevalence. Circulation 142:184-186

13. Qureshi Al, Baskett WI, Huang W et al (2021) Acute ischemic stroke and COVID-19: an analysis of 27 676 patients. Stroke 52:905-912

14. Ramacciotti $E$, Macedo AS, Biagioni RB et al (2020) Evidence-based practical guidance for the antithrombotic management in patients with Coronavirus disease (COVID-19) in 2020. Clin Appl Thromb Hemost 26:1076029620936350

15. Tang N, Li D, Wang X et al (2020) Abnormal coagulation parameters are associated with poor prognosis in patients with novel coronavirus pneumonia. JThromb Haemost 18:844-847

16. Wagner WL, Hellbach K, Fiedler MO et al (2020) Microvascular changes in COVID-19. Radiologe 60:934-942

17. Wang D, Hu B, Hu C et al (2020) Clinical characteristics of 138 hospitalized patients with 2019 novel Coronavirus-infected pneumonia in Wuhan, China. JAMA 323:1061-1069

18. Wichmann D, Sperhake JP, Lutgehetmann M et al (2020) Autopsy findings and venous thromboembolism in patients with COVID-19: a prospective cohort study. Ann Intern Med 173:268-277

19. Zhang R, Ni L, Di X et al (2021) Systematic review and meta-analysis of the prevalence of venous thromboembolic events in novel coronavirus disease-2019 patients. J Vasc Surg Venous Lymphat Disord 9:289-298e5

\section{SpringerMedizin.de Lesen Sie Ihre Fachzeitschrift auch als ePaper!}

Als Abonnent können Sie Ihre Zeitschrift in verschiedenen Formaten lesen. Wählen Sie je nach Vorliebe und Situation aus, ob Sie die Zeitschrift als Print-Ausgabe, in Form von einzelnen Beiträgen auf springermedizin.de oder aber als komplette, elektronische ePaper-Ausgabe lesen möchten.

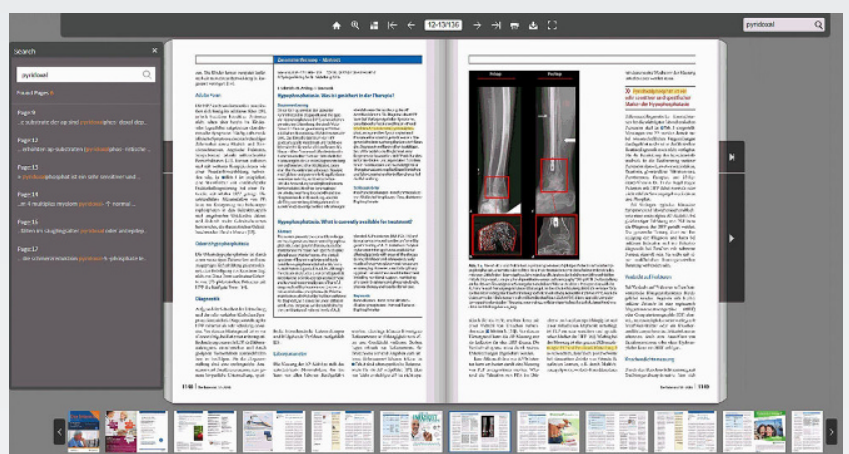

Die ePaper sind die identische Form der gedruckten Ausgaben. Sie sind nutzbar auf verschiedenen Endgeräten wie PC, Tablet oder Smartphone

Das sind die Vorteile des ePapers:

$>$ Das verlinkte Inhaltsverzeichnis führt Sie direkt zum gewünschten Beitrag

> Eine Suchfunktion ermöglicht das Auffinden von Schlagworten innerhalb der Zeitschrift.

> Jede Ausgabe kann als PDF heruntergeladen und damit auch offline gelesen werden bzw. auch gespeichert oder ausgedruckt werden.

> Als Abonnent haben Sie Zugang zu allen ePaper-Ausgaben ab 2016.

Sie finden die ePaper auf SpringerMedizin.de bei der jeweiligen Ausgabe Ihrer Fachzeitschrift. Klicken Sie auf den Button „Ausgabe als ePaper lesen“. 